\title{
Secondary Stroke After Thrombolytic Administration; A Case Report
}

\author{
Farzad Ashrafi $^{1,2}$; Alireza Baratloo $^{3}$; Zahra Nasiri $^{1, *}$; Maryam Soleymani ${ }^{1}$; Sina Asaadi ${ }^{4}$ \\ ${ }_{1}^{1}$ Department of Neurology, Shohadaye Tajrish Hospital, Shahid Beheshti University of Medical Sciences, Tehran, IR Iran \\ ${ }^{2}$ Functional Neurosurgery Research Center, Shohadaye Tajrish Hospital, Shahid Beheshti University of Medical Sciences, Tehran, IR Iran \\ ${ }_{3}^{3}$ Department of Emergency Medicine, Shohadaye Tajrish Hospital, Shahid Beheshti University of Medical Sciences, Tehran, IR Iran \\ ${ }^{4}$ Department of Medicine, Shahid Beheshti University of Medical Sciences, Tehran, IR Iran \\ ${ }^{*}$ Corresponding author:Zahra Nasiri, Department of Neurology, Shohadaye Tajrish Hospital, Shahid Beheshti University of Medical Sciences, Tajrish Square, Tehran, IR Iran. Tel: +98- \\ 9126237429, Fax:+98-2122721155, E-mail: missz.nasiri@yahoo.com \\ Received: January 4, 2014; Revised: March 5, 2014; Accepted: March 30, 2014
}

\begin{abstract}
Introduction: Ischemic stroke is still a leading cause of death and long-term disability all around the world. Restoration of blood flow with thrombolytic agents like tissue plasminogen activator (t-PA) is the only way that may rescue patients exposed to cerebral ischemia. Complications of these agents are commonly related to hemorrhage, arterial reocclusion, anaphylaxis, or reperfusion damage. Recurrence of stroke in the same or other cerebral arteries only rarely causes early deterioration during or shortly after thrombolytic administration. We report a patient who experienced a second stroke despite intravenous thrombolytic therapy.

Case Presentation:A68-year-old man with the history of uncontrolled hypertension and diabetes mellitus presented with acute ischemic stroke in the territory of vertebrobasilar system to the emergency department. Atrial fibrillation (AF) rhythm, high blood sugar and uncontrolled hypertension were recorded on admission. Echocardiography performed before thrombolysis, did not identify any embolic sources. Five Hours after intravenous injection of tissue Plasminogen Activator (t-PA), the patient became comatose and developed a recurrent infarction in the territory of right middle cerebral artery (MCA).

Discussion: It seems that thrombolysis therapy in patients with the risk factors of recurrent stroke, must be done with more preparation and readiness for concurrent processing. Regardless of normal trans-thoracic echocardiography, in patients with AF rhythm, transesophageal echocardiography may be helpful. Also performing appropriate randomized trial to compare the incidence of recurrent stroke in patients under t-PA therapy and controls were still needed.
\end{abstract}

Keywords:Stroke; Atrial Fibrillation; Tissue Plasminogen Activator

\section{Introduction}

Ischemic stroke is still a leading cause of death and long-term disability all over the world (1). Restoration of blood flow to the ischemic brain as early as possible is the only way to rescue patients exposed to cerebral ischemia. As a result of the National Institute of Neurological Disorder and Stroke (NINDS) Tissue Plasminogen Activator (t-PA) Stroke Study Group Trial, the U.S. Food and Drug Administration (FDA) approved intravenous recombinant t-PA for treatment of ischemic stroke within three hours of onset (2).

Complications of intravenous t-PA for acute ischemic stroke are commonly related to hemorrhage, arterial reocclusion, anaphylaxis, or reperfusion damage. However, with the increased use of t-PA for acute ischemic stroke, many serious complications that are directly or indirectly related to t-PA administration have been reported more frequently. Recurrence of embolism in the same or other cerebral territory rarely reported during or shortly after thrombolysis may call for future studies to improve current guidelines. We report a patient who experienced recurrent embolism after intravenous (IV) thrombolytic injection.

\section{Case Presentation}

A 68-year-old man with the sudden loss of consciousness was admitted to our emergency department 45 minutes after the onset of symptoms. Physical examination on admission revealed a blood pressure of 160/90 mm Hg and an irregular heart rate of 110 beats/minute. He had a history of uncontrolled hypertension for about two years and diabetes mellitus type 2 since five years ago. He was somnolent and exhibited dense right hemiplegia. His NIHSS (National Institutes of Health Stroke Scale) score was 15 . His blood glucose was $320 \mathrm{mg} / \mathrm{dL}$, and baseline electrocardiography (ECG) showed AF rhythm with no evidence of ischemia. Echocardiography also confirmed AF rhythm. Levels of creatine kinase MB (CKMB) and tro

Implication for health policy/practice/research/medical education:

Complications of intravenous tissue plasminogen activator (t-PA) for acute ischemic stroke are commonly related to hemorrhage, arterial reocclusion, anaphylaxis, or reperfusion damage. However, with the increased use of t-PA for acute ischemic stroke, many serious complications of t-PA have been reported. Recurrence of embolism in the same or other cerebral arteries only rarely causes early deterioration during or shortly after thrombolysis. We report a patient who experienced recurrent embolism after intravenous thrombolytic injection.

Copyright (C) 2015, Tehran University of Medical Sciences. This is an open-access article distributed under the terms of the Creative Commons Attribution License, which permits unrestricted use, distribution, and reproduction in any medium, provided the original work is properly cited. 


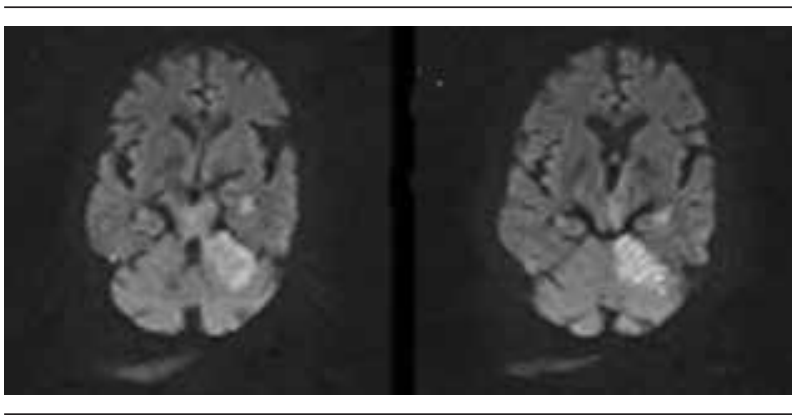

Figure 1. Diffusion-Weighted MRI Images one Hour After Admission in Emergency Department

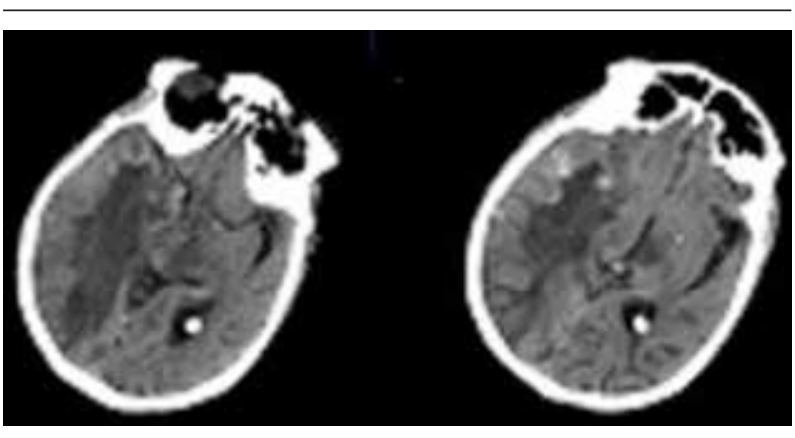

Figure 2. CT Scan 24 Hours After t-PA Administration

ponin I (TnI) were normal. Brain computed tomography (CT) scan on the first hour of admission disclosed no ischemic changes. Diffusion-weighted magnetic resonance imaging (MRI) revealed acute ischemic stroke in the territory of vertebrobasilar system and evidence of infarction in the brainstem too (Figure 1).

Patient was treated with IV t-PA; $0.9 \mathrm{mg} / \mathrm{kg}, 10 \%$ bolus, $90 \%$ continuous infusion over 60 minutes, which started 130 minutes after the onset of symptoms in emergency department. Seven hours after beginning of symptoms, brain CT scan showed an infarct in the territory of vertebrobasilar system. However, 24 hours after t-PA administration, the patient became comatoseal. Both pupils were $6 \mathrm{~mm}$ dilated and fixed in mid-position with no light reflexes. He developed quadriplegia and positive Babinski reflex on both sides. His NIHSS score became 40. Emergency brain CT scan showed no hemorrhagic changes but acute infarction in the territory of right middle cerebral artery (MCA) (Figure 2). Furthermore, patient's brain edema became gradually worse.

Treatment started with mannitol, aspirin, atorvastatin, and diprydamole. Patient was admitted in Intensive Care Unit (ICU) because of deep coma and after 10 days, he died because of cardiopulmonary failure.

\section{Discussion}

Recurrent ischemic stroke is defined as the occurrence of new neurological symptoms that suggests involvement of unaffected vascular territories and evidence of correspond- ing ischemic lesions on brain CT scan. This phenomenon has been reported in rare cases secondary to mechanical disruption. In another study, it was reported during intravenous thrombolysis secondary to atrial fibrillation. Yet in another case, a patient with history of cardiomyopathy developed recurrent stroke after IV t-PA injection because of cardioembolic stroke (3-5). It should be mentioned that correct brain imaging interpretation is the basic medical education for physicians who handle such patients in emergency departments or critical care units (6).

Our patient as we mentioned above, had several clinical risk factors of ischemic stroke such as AF rhythm, diabetes mellitus and hypertension. Among these risk factors, AF rhythm can increase the risk of stroke approximately six times (7). Although, neither proved, nor rejected, the stroke may be caused by cardiogenic embolism, given that he had uncontrolled atrial fibrillation and no evidence indicating another embolic source. So in spite of normal transthoracic echocardiography, in patients with $\mathrm{AF}$, transesophageal echocardiography may be mandatory to find the possible source of the embolus. Obviously, disintegration of thrombi can occur spontaneously and is not necessarily associated with t-PA administration. Still, the fact that neurological deterioration occurred after t-PA initiation strongly argues for a causative role of t-PA(4).

Although predictor factor for recurrent embolism due to thrombolytic injection remains unclear in our patient, as well as in the aforementioned cases, a mural thrombus in the left atrium, which was seen in the previously reported cases with recurrent stroke might be a risk factor for recurrent embolism $(5,8)$. On the other hand, performing echocardiography to detect mural thrombus before thrombolysis is difficult, given the very short therapeutic time window for thrombolytic usages.

It seems that thrombolysis therapy in patients with risk factors that predispose a secondary stroke must be done with more preparation and readiness for concurrent processing. For instance, even with normal and available transthoracic echocardiography, in patients with AF rhythm, transesophageal echocardiography may be more helpful. Also performing appropriate randomized trial to compare the incidence of recurrent stroke in the patients under t-PA therapy with controls can help us have a better understanding about risk factors of recurrent stroke and it's complications that can affect management of recurrent stroke.

\section{Acknowledgements}

We would like to thank all the emergency department personnel who helped in diagnosis and management of this patient.

\section{Authors' Contributions}

Study supervision: Farzad Ashrafi; Study concept and design, critical revision of the manuscript: Alireza Baratloo, Zahra Nasiri; Acquisition of data: Maryam Soleymani, and;Manuscript Drafting: Sina Asaadi. 


\section{References}

1. Sander D, Carolei A, Diehm C, Hennerici MG, Rothwell PM. Challenges to the management of high-risk stroke patients with multiple-site occlusive vascular disease. Cerebrovasc Dis. 2011;31(4):315-21.

2. Wardlaw JM, Murray V, Berge E, del Zoppo G, Sandercock P, Lindley $\mathrm{RL}$, et al. Recombinant tissue plasminogen activator for acute ischaemic stroke: an updated systematic review and meta-analysis. The Lancet. 2012;379(9834):2364-72.

3. Shirahama K, Inatomi Y, Yonehara T, Hirano T, Uchino M. Recurrence of embolic stroke during intravenous thrombolysis.J Stroke Cerebrovasc Dis. 2012;21(1):84-6.

4. Sposato LA, Salutto V, Beratti DE, Monti P, Riccio PM, Mazia C. Adverse outcome of early recurrent ischemic stroke secondary to atrial fibrillation after repeated systemic thrombolysis. Case Rep Vasc Med. 2013;2013:371642.
5. Awadh M, MacDougall N, Santosh C, Teasdale E, Baird T, Muir KW. Early recurrent ischemic stroke complicating intravenous thrombolysis for stroke: incidence and association with atrial fibrillation. Stroke. 2010;41(9):1990-5.

6. Arhami Dolatabadi A, Baratloo A, Rouhipour A, Abdalvand A, Hatamabadi $\mathrm{H}$, Forouzanfar M, et al. Interpretation of Computed Tomography of the Head: Emergency Physicians versus Radiologists. Trauma Mon. 2013;18(2):86-9.

7. Mori M, Naganuma M, Okada Y, Hasegawa Y, Shiokawa Y, Nakagawara J, et al. Early neurological deterioration within 24 hours after intravenous rt-PA therapy for stroke patients: the Stroke Acute Management with Urgent Risk Factor Assessment and Improvement rt-PA Registry. Cerebrovasc Dis. 2012;34(2):140-6.

8. Kwon TH, Kim BM, Nam HS, Kim YD, Heo JH, Kim DI, et al. Carotid stenting in acute ischemic stroke patients with intraluminal thrombus. Neuroradiology. 2011;53(10):773-8. 